\title{
EDITORIAL
}

\section{Resection rates in lung cancer patients}

\author{
K. Moghissi*, C.K. Connolly**
}

The first pneumonectomy for cancer performed by GRAHAM and Singer [1] in 1933 was an important milestone in thoracic surgery and established surgical resection as the primary method of treating carcinoma of the lung. Since then advances in diagnostic methods, progress in surgical techniques and developments in anaesthesia, intensive care and perioperative management have contributed to the expansion of surgical indication.

Paradoxically, however, only a small proportion of lung cancer sufferers are either referred to or undergo resectional surgery as shown in the article by DAMHUIS and Schutte [2] in this issue of the Journal. In fact the $20 \%$ resection rate in a series of 7,899 patients from the Rotterdam Region reported by the authors represents the average of many European countries but is above that of the UK. In the UK, some 32,500 patients are diagnosed annually as having lung cancer [3] and the 1994 thoracic surgery register compiled by the Society of Cardiothoracic Surgeons of Great Britain and Ireland indicated 3,333 resections, giving a "calculated" resection rate of $10 \%$. In the Yorkshire region of Great Britain where there is a comprehensive cancer register over recent years $300-500$ of about 3,000 cancers have been resected, giving a constant rate of $10-12 \%$ albeit against a slowly increasing age at presentation [4]. Thus, the UK rates at $10-12 \%$ are even lower than the European rate at $20 \%$.

The issue of why only 10-20\% of all lung cancer sufferers undergo resectional surgery could not be addressed in the article by Damhuis and Schutte [2] but nor it seems has this matter been considered elsewhere in spite of the fact that: 1) lung cancer is the commonest malignant tumour in the western world and 2) surgical operation appears to be the only treatment modality which offers hope of cure for all but a few subjects with good prognosis small cell cancer.

Given the present climate of audit and accountability, this issue should be addressed and the following relevant questions asked. Is it really true that when they have their first symptom, twice as many patients are operable in Europe as compared with the UK? If so, why? Is it because the disease is different or co-morbidity is a much greater problem? Is it really true that only up to $20 \%$ of patients are operable at presentation and is this because patients reach surgeons too late after their first symptoms or is it because the selection criteria are too restrictive?

*Correspondence: K. Moghissi, BUPA Hospital Hull and East Riding, Anlaby, Hull, N. Humberside HU10 7AZ, UK. **Friargate Hospital, Northallerton, N. Yorkshire, UK.
Many surgeons do indeed hold the view that too few patients reach the surgical unit and that those who do are referred at a stage that might with more appropriate management be less advanced. Whether the potential effect of delay proves to be large or small it must be remembered that all patients, particularly those whose survival cannot be modified, will benefit from appropriate management of their symptoms at the earliest opportunity. The extent of this problem requires investigating and defining.

Even now, with the present evidence, much can be done to educate potential patients, those involved in primary care and the specialists who first see the patients. The work of DAmHUIS and SchutTe [2] and data from the literature show that age need no longer present a barrier $[5,6,7]$. Admittedly, for individuals aged $70 \mathrm{yrs}$ and over the risk of operation may be higher particularly when it involves pneumonectomy. However, with appropriate selection it is possible to have an acceptable postoperative mortality, as shown by DAMHUIS and SCHUTTE [2]. Although postoperative morbidity is generally higher than similar operations for younger patients, 2 year survival is now independent of age in Yorkshire [4].

Generalists and nonsurgical oncologists should remember that the contribution of the affected lung to lung function is likely to be reduced and so the potential effect of surgery on lung function overestimated, particularly when parenchyma-saving conservative techniques can be applied. They may not fully appreciate the role of surgery in selected patients with stage III cancer, either as T3$\mathrm{T} 4$ or $\mathrm{N} 2$ disease at certain isolated stations.

In the final analysis, achieving the optimal operability rate will depend on three factors:

1. As it is generally acknowledged that screening is not helpful, appropriate and expeditious management of the disease from the first symptom is essential.

2. Participation of the surgeon in the judgement of operability versus inoperability. This is impossible if physicians or oncologists only refer to surgeons in anticipation of operation. It is essential that all patients, except possibly those of worst prognosis, should benefit from the judgement of a multidisciplinary team which will include a surgeon who must be responsible for the criteria of inoperability and unresectability, as it is his team which has the responsibility of performing the operation. Unless he sees a proportion of inoperable patients, he can never draw the correct line between inoperability and operability and adjust it with time.

3. Full advantage must be taken of any increase in surgical rate which might be achieved by downstaging with 
adjuvant chemotherapy. Although not yet of proven advantage the signs are encouraging and this important issue should be addressed in the near future by a multicentre trial on a large number of patients with a carefully planned protocol.

\section{References}

1. Graham EA, Singer JJ. Successful removal of an entire lung for carcinoma of the bronchus. J Am Med Assoc 1933; 101: 1371-1373.

2. Damhuis RAM, Schutte PR. Resection rates and postoperative mortality in 7,899 patients with lung cancer. Eur Respir J 1995; 9: 8-11.
3. Management of lung cancer. Current clinical practice. Standing medical advisory committee report of working group, chaired by Prof. J.M.A. Whitehouse, 1994, p. 1.

4. Joslin C, Rider L (eds). Cancer in Yorkshire. 1. Lung Cancer. Yorkshire Regional Cancer Organisation, Leeds, May 1993.

5. Roxbrugh JC, Thompson J, Goldstraw P. Hospital mortality and long-term survival after pulmonary resection in the elderly. Ann Thorac Surg 1991; 51: 800-803.

6. Patel RL, Townsend ER, Fountain SW. Elective pneumonectomy: factors associated with morbidity and operative mortality. Ann Thorac Surg 1992; 54: 84-88.

7. Gebitekin C, Gupta NK, Martin PG, Saunders NR, Walker DR. Long-term results in the elderly following pulmonary resection for non-small lung carcinoma. Eur $J$ Cardiothorac Surg 1993; 7: 653-656. 